\title{
Independência efetora e prática moderada com a mão esquerda em destrímanos
}

\author{
Ulysses O. Araújo \\ Cássio M. Meira Jr. \\ Jaqueline F.O. Neiva \\ Miriam Y. Umeki
}

\author{
Laboratório de Comportamento Motor \\ Escola de Educação Física e Esporte \\ Universidade de São Paulo \\ Brasil
}

https://doi.org/10.5628/rpcd.08.02.251

\section{RESUMO}

A independência efetora (capacidade de executar uma tarefa com diferentes efetores) foi investigada com foco na transferência bilateral de aprendizagem. Especificamente, foi investigada a hipótese de que a prática com a mão esquerda em destrímanos levasse a aprendizagem independente de efetor seguida de aprendizagem dependente de efetor, de forma similar à caracterizada na prática com a mão direita. Dezoito participantes adultos (idade média $25.6 \pm 2.9$ anos) realizaram vinte tentativas divididas em dois dias numa tarefa de destreza manual fina (inserção de pinos - Grooved Pegboard Test), inserindo 25 pinos por tentativa. Foram realizados um pré-teste, um teste de retenção intermediário após cinco tentativas e um teste de retenção final após o término da prática. Os resultados corroboraram a hipótese: o desempenho da mão esquerda melhorou do pré-teste ao teste intermediário, e deste para o teste final, enquanto o desempenho da mão direita apresentou melhora apenas do pré-teste para o teste intermediário. O padrão de independência efetora seguida de especificidade com relação ao efetor utilizado foi similar ao relatado na prática com a mão direita em destrímanos. Sugere-se, portanto, uma reconsideração da independência absoluta de efetor ao longo da prática.

Palavras-chave: independência efetora, transferência bilateral, prática, aprendizagem motora

\section{ABSTRACT \\ Effector independence and moderate practice with the left hand in right-handers}

The effector independence (ability to perform a task using different effectors) was investigated focusing the bilateral transfer of learning. Specifically, we investigated the hypothesis that practice using the left hand in right-handers would lead to effector independent learning followed by effector dependent learning, as addressed in the findings with practice using the right hand. Eighteen adults (mean age $25.6 \pm 2.9$ years) performed twenty trials in a fine hand dexterity task (pin insertion) over two days (Grooved Pegboard Test), inserting 25 pins per trial. The design consisted of a pre-test, an intermediate retention test after five trials, and a final retention test after the completion of the practice. The results supported the hypothesis: performance of the left hand improved from the pre-test to the intermediate retention test, and from the latter to the final retention test, while the performance of the right hand improved only from the pre-test to the intermediate retention test. The pattern of effector independence followed by specificity regarding the effector was similar to the one found over practice with the right hand in right-handers. This suggests a reconsideration of an absolute effector independence throughout practice.

Key- words: effector independence, bilateral transfer, practice, motor learning 


\section{INTRODUÇÃOO}

A transferência de aprendizagem pode ser definida como o ganho na capacidade de desempenho em uma tarefa como resultado da prática ou experiência em outra tarefa(23). Recentemente, o foco em estudos de transferência de aprendizagem tem se voltado à transferência de efetor, situação na qual a capacidade de desempenho com um efetor específico varia em função da prática da mesma tarefa com outro efetor. Mais especificamente, o aprendizado é considerado dependente de efetor na medida em que a aprendizagem com um conjunto de músculos não é genérica em relação a outros conjuntos(18) e independente de efetor quando a transferência para outros grupos musculares acontece. Podemos imaginar uma situação onde a independência efetora é mais evidente ao escrever: é possível utilizar ambas as mãos ou mesmo os pés para manusear o instrumento de escrita, e apesar das diferenças na execução com os diversos efetores ainda é possível reconhecer um padrão distinto de letras e palavras.

Além de implicações práticas, como no delineamento de programas de intervenção em que haja aprendizagem de habilidades motoras, há implicações teóricas na transferência, tanto em geral como na transferência específica de efetor. Diversas proposições teóricas correntes têm como uma de suas predições certa independência efetora. A aquisição de uma representação mental de uma classe de habilidade em um nível relativamente abstracto é uma das proposições da Teoria de Esquema (24), que permitiria a execução dos movimentos dessa classe de acordo com as demandas ambientais.

A hipótese que o aprendizado é representado por uma regra abstracta - descrita em termos de equações de movimento para um determinado nível de descrição - que governa como estados coordenativos se apresentam e evoluem em função de diferentes tipos de restrições é formulada pelos defensores da dinâmica da coordenação (coordination dynamics) $(11,25)$. Para os autores, as mudanças na dinâmica intrínseca relativas ao aprendizado seriam transferíveis para diferentes efetores, desde que respeitadas as diferenças biomecânicas e anatómicas entre membros, pois toda essa dinâmica, responsável pela emergência dos padrões de coordenação, seria alterada no processo. Predições em que a representação da acção possui características abstractas, relativamente independentes da execução do movimento, também fazem parte de outras proposições teóricas $(12,15,22)$. Contudo, apesar do grande suporte empírico à hipótese de certa independência efetora, há duvidas quanto aos limites desta independência( ${ }^{(8)}$.

Uma forma específica de investigar a independência de efetor é através da transferência bilateral de aprendizagem, definida como a transferência da aprendizagem da execução de um movimento com um membro para o membro no lado oposto(21). Diversos estudos apontam transferência positiva entre membros para diversas tarefas, desde as pesquisas iniciais utilizando escrita espelhada ${ }^{(4)}$ e rotor de perseguição(1) até as mais recentes, com tarefas de tempo de reacção seriado(7), aprendizagem de padrões rítmicos multiarticulares ${ }^{(3)}$ e tarefas sincronizatórias de diferentes complexidades(27).

Embora pouco se questione actualmente a ocorrência de transferência em alguma medida para o membro oposto, assim como na investigação sobre independência efetora, estudos recentes sugerem um componente específico para o membro utilizado na prática ${ }^{(30,31)}$.

Além disso, outros autores sugerem uma seqüência em que ganhos iniciais, independentes de efetor, dão lugar a um aprendizado específico ao efetor utilizado após um período mais prolongado de prática. Esse padrão foi apontado inicialmente por Bapi, Doya e Harner(2) na transferência entre diferentes grupos musculares, não contralaterais. Os autores investigaram o aprendizado de uma sequência composta por apertos sucessivos de teclas com os dedos, em resposta a estímulos visuais. A transferência para condições nas quais havia rotação de 90 graus na posição dos dedos, do estímulo visual ou de ambos foi investigada no estágio inicial, intermediário e avançado de prática. Os resultados apontaram para uma dependência efetora no estágio avançado de prática e corroboraram, segundo os autores, a existência de uma representação específica para efetor adquirida de forma relativamente lenta.

Nos estudos cujo foco é a transferência para o membro contralateral, com utilização de testes nos estágios intermediários da prática, o padrão de ganhos independentes de efetor seguidos de ganhos dependentes de efetor também tem sido relatado. Park e 
Shea(19) investigaram a transferência para o membro contralateral e para o grupo muscular antagonista em relação ao utilizado na prática numa tarefa de produção de um padrão ondulatório de força, após 1 dia (200 tentativas) e 4 dias (800 tentativas) de prática. O desempenho em ambos os testes de transferência não foi melhor após 4 dias de prática do que apenas após 1 dia, apesar de ter havido uma melhora significativa no teste de retenção. Park e Shea(20) abordaram novamente o problema utilizando uma tarefa com estrutura relativamente mais complexa, a aprendizagem de movimentos utilizando uma alavanca em direcção a uma sequência determinada de alvos. O desempenho do membro contralateral (teste de transferência) foi similar ao do membro empregado na aprendizagem (teste de retenção) após 1 dia de prática (experimentos 1 e 2). Contudo, o desempenho no teste de transferência foi inferior ao desempenho no teste de retenção após 4 dias de prática (experimento 2). Para os autores, isto sugere que o processo que leva à consolidação da execução da resposta leva também à utilização de informação específica em relação ao membro utilizado, o que explicaria a degradação do desempenho na realização da tarefa com o membro contralateral.

Vangheluwe, Suy, Wenderoth e Swinnen(28) obtiveram resultados que apontam na mesma direção, ao investigar a aquisição de um padrão de coordenação bimanual. Ainda que neste tipo de tarefa um dos membros não esteja em repouso enquanto o outro pratica, e desse modo o estudo não possa ser considerado estritamente de transferência bilateral, é relevante notar a semelhança dos resultados obtidos. Os autores investigaram a aquisição de um padrão em que um dos braços se movia em uma frequência duas vezes mais rápida que o outro (2 para 1$)$, sendo que para um dos grupos o braço mais rápido era o direito e para o outro o esquerdo. Após um período de prática (160 tentativas em 4 dias), os participantes foram solicitados a executar o padrão inverso (1 para 2) em relação ao padrão anteriormente realizado, isto é, com o braço anteriormente mais lento agora mais rápido, por mais 4 dias. Apesar da melhora do pré-teste para um teste intermediário na configuração praticada (2 para 1), o desempenho piorou no mesmo período para a configuração não praticada (1 para 2). Além disso, a melhora do desempenho do teste intermediário para o pós-teste, que utilizou uma configuração contrária à inicial, teve influência negativa no padrão aprendido anteriormente, de modo que o erro no pós-teste para essa configuração foi superior ao do teste intermediário. Para os autores, os resultados não apoiam pontos de vista correntes que enfatizam a independência efetora, e sugerem a necessidade de considerar que a representação dos movimentos na memória inclui tanto aspectos abstractos, transferíveis a outros efetores, quanto aspectos específicos e não transferíveis.

Estudos investigando a transferência bilateral de aprendizagem ao longo da prática têm sido realizados, na maior parte dos casos, com a mão preferida. Para a maior parte da população, a preferência manual em diversas tarefas é pelo lado direito. Para a população manualmente destra, evidências obtidas com técnicas de visualização da actividade cerebral associam algumas áreas (especialmente no hemisfério cerebral esquerdo) previamente relacionadas ao controle de movimentos tanto com o lado preferido quanto com o lado não preferido como responsáveis também pela aprendizagem de sequências de movimentos com ambas as mãos ${ }^{(5)}$. Assim, é possível presumir que caso a aprendizagem envolva substratos neurais de forma similar para movimentos utilizando tanto a mão esquerda como a direita, um padrão similar de independência seguida de dependência de efetor será observado também na utilização da mão não preferida.

Karni et al. $(9,10)$ e Korman, Raz, Flash e Karni(13) são dos poucos estudos que investigam a independência de efetor na prática com a mão não-preferida. Estes estudos investigaram a aquisição de uma sequência de toques entre dedos em um período de prática relativamente longo: cinco sessões de prática ao longo de aproximadamente duas semanas(13) ou dez sessões de prática ao longo de três semanas $(9,10)$. A melhora dos participantes após a prática em uma sequência de toques entre os dedos não trouxe aumento no desempenho nem em uma sequência similar executada com a mão utilizada na prática, nem na mesma sequência quando realizada com a mão contralateral $(9,10)$. Num padrão similar ao observado na utilização da mão direita em destros, os ganhos da primeira sessão de prática, realizada 
com a mão esquerda, se transferiram quase completamente para a mão direita; contudo, o mesmo não ocorreu após quatro outras sessões de prática(13). Embora, no seu conjunto, os resultados dos estudos de Karni et al. $(9,10)$ e Korman et al.(13) apontem para um padrão de independência seguido de dependência de efetor para a prática realizada com a mão não preferida por destros, apenas o estudo de Korman et al.(13) utilizou testes de transferência para a mão contralateral em estágios intermediários da prática. Além disso, a sequência de ganhos independentes seguidos de ganhos dependentes nesses estudos foi observada ao longo da prática realizada ao longo de uma escala de tempo relativamente longa, enquanto a mesma seqüência de ganhos foi observada após alguns dias quando a prática foi realizada com a mão direita em destros $(19,20)$. A questão que se coloca é: a sequência de independência/dependência de efetor na prática com a mão esquerda, em destros, pode ser observada em uma escala de tempo similar à da prática com a mão direita?

Com o objectivo de preencher esta lacuna, o presente estudo visa investigar a transferência bilateral (intermanual) de aprendizagem da prática realizada com a mão não preferida em participantes manualmente destros, com a utilização de testes de transferência para a mão contralateral nos estágios inicial e intermediário da prática. Considerando também o padrão relativamente robusto da sequência de ganhos independentes seguidos de ganhos dependentes de efetor, é razoável supor que um padrão similar possa ser observado e surja após poucos dias de prática, como em outros estudos relatados cuja prática foi realizada com a mão preferida.

\section{MATERIAL E MÉTODOS}

\section{Participantes}

Participaram do estudo 18 voluntários adultos, com idade média de 25,6 $( \pm 2,9)$ anos, sendo 9 participantes do sexo masculino e 9 do sexo feminino. $O$ Inventário de Edimburgo foi utilizado para determinação da preferência manual (17). Os participantes possuíam forte preferência pela mão direita, e não apresentavam transtornos motores evidentes. Ademais, possuíam visão normal ou corrigida para normal e não possuíam experiência na tarefa. $\mathrm{O}$ consentimento informado foi obtido antes do início da participação. Os participantes foram tratados de acordo com a Declaração de Helsinquia.

\section{Instrumento}

Foi utilizado como instrumento o Grooved Pegboard Test (Lafayette Instrument Company, modelo $n^{\circ}$ 32025). O Grooved Pegboard é um teste de destreza manual composto por uma superfície com 25 orifícios, cada um possuindo uma reentrância posicionada em diferentes direcções, e uma superfície côncava onde são colocados pinos. Os pinos também possuem uma reentrância, que deve coincidir espacialmente com a do orifício para que haja encaixe. O objectivo da tarefa era preencher os 25 orifícios no menor tempo possível, segurando e inserindo apenas um pino por vez. O tempo para realização da tarefa foi utilizado como medida de desempenho, sendo mensurado com um cronómetro digital, arredondado para o $0,1 \mathrm{~s}$ mais próximo, registado e fornecido como conhecimento de resultado aos participantes em todas as tentativas de prática. Todavia, esta informação não foi fornecida aos participantes após a realização dos testes.

\section{Delineamento e procedimentos experimentais}

As etapas do experimento foram realizadas na mesma ordem para todos os participantes. Após a explicação da tarefa foi feito um procedimento de familiarização, com a mão não preferida $(E)$ e em seguida com a mão preferida (D). Na familiarização o participante preenchia a primeira linha do Pegboard, ou seja, os primeiros cinco pinos da linha mais distante. Em seguida foi realizado o pré-teste (PT), no qual o participante deveria preencher todos os 25 orifícios do Pegboard. Após o pré-teste, o participante iniciou a prática. Todas as tentativas de prática foram realizadas com a mão não preferida $(E)$. Foram realizadas cinco tentativas no primeiro dia de prática, com a inserção dos 25 pinos em cada uma delas. Após as tentativas, foi dado um intervalo não activo de cinco minutos e foi realizado o primeiro teste de retenção (RT1). Após o teste de retenção, o sujeito praticou a tarefa por mais cinco tentativas. No segundo período de colecta, dois dias depois do primeiro, o participante realizou novamente a familiarização da tarefa, seguida de uma nova sessão de prática, com a execução de dez tentativas. Depois de um intervalo inactivo de cinco minutos, o participan- 


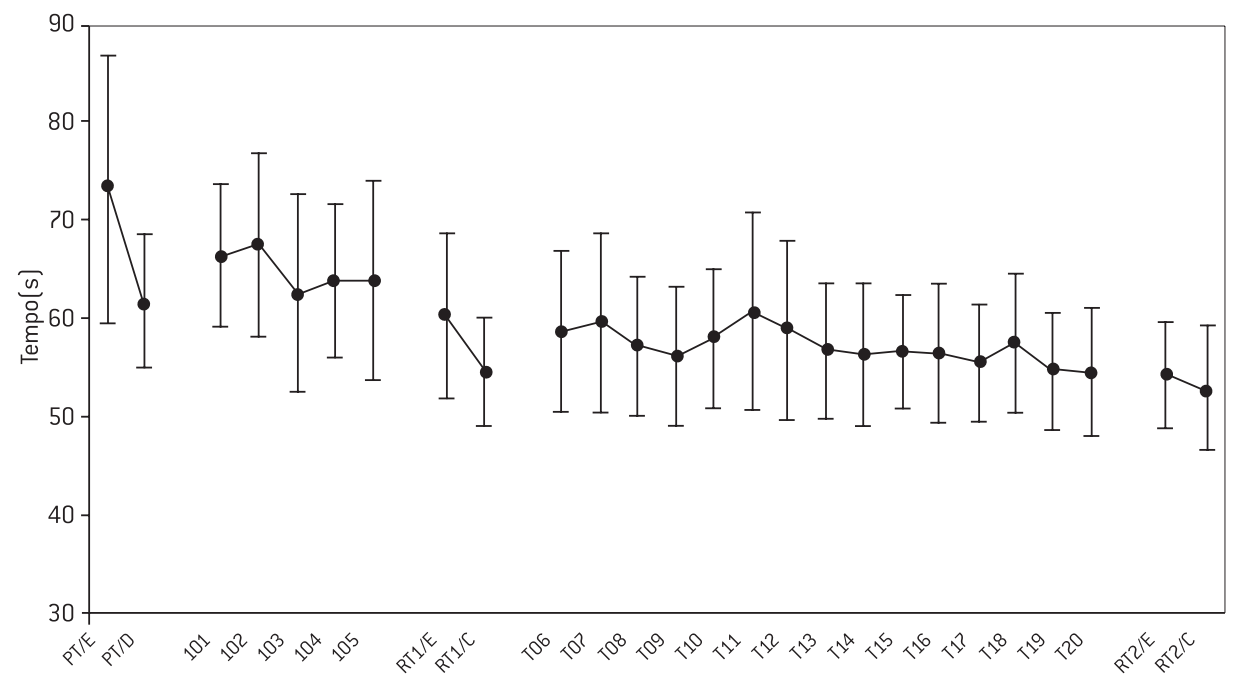

Figura 1. Média e desvio padrão do desempenho por tentativa/teste.

te realizou o segundo teste de retenção (RT2). Em função dos objectivos do estudo, todos os testes (PT, RT1 e RT2) foram realizados com ambas as mãos, sempre iniciando pela mão não preferida (E). Em todas as etapas do experimento, quando a tarefa foi executada com a mão esquerda os pinos deveriam ser inseridos da direita para a esquerda, iniciando pela linha mais distante do participante até a mais próxima. Na utilização da mão preferida (D), o participante deveria iniciar a linha pelo lado esquerdo.

\section{Medidas e Análise}

A variável dependente do estudo foi o tempo, medido em segundos. Considerou-se, na análise, apenas os resultados obtidos nas tentativas referentes aos testes (PT, RT1 e RT2). De primeiro, estes dados foram submetidos a uma análise estatística exploratória (análise descritiva, checagem de outliers, testes de normalidade de distribuição e de homogeneidade de variâncias). Os dados foram ajustados relativamente às observações definidas como outliers extremos. Pelo fato de não ter havido violações de normalidade de distribuição dos dados e heterogeneidade de variâncias, os dados referentes aos testes foram submetidos a análises de variância a dois factores (lado utilizado x prática) com medidas repetidas no segundo factor. Na localização das diferenças, o teste post-hoc de Bonferroni foi utilizado.

\section{RESULTADOS}

O desempenho nas diferentes fases do experimento é apresentado na Figura 1. É possível observar uma tendência geral de queda no tempo necessário para realizar a tarefa ao longo das tentativas de prática. Também é possível observar a diminuição das diferenças entre o desempenho na realização da tarefa com a mão esquerda, empregada na prática, e a mão direita, não empregada.

Detectou-se efeito nos factores principais "lado utilizado" $[\mathrm{F}(1,32)=6.80, \mathrm{p}<0.05]$, "prática" $[\mathrm{F}(2,64)$ $=53.16, \mathrm{p}<0.001]$ e na interacção entre ambos os factores $[\mathrm{F}(2,64)=6.47, \mathrm{p}<0.05]$. Testes post-hoc localizaram as diferenças na execução com a mão não preferida ao longo da prática em todos os testes: o desempenho foi melhor em RT1/E e em RT2/E, em comparação a PT/E ( $p<0.001$ para ambas as comparações); ainda, em RT2/E o desempenho foi superior a RT1/E ( $<0.05)$. Para a mão preferida, porém, as melhoras relacionadas à prática se restringiram ao período inicial: em RT1/D e RT2/D os desempenhos foram superiores quando confrontados com PT/D ( $p<0.007$ e $p<0.001$ respectivamente). Foram localizadas, ainda, diferenças no desempenho entre a utilização da mão preferida e da mão não preferida no teste inicial (PT/E inferior a PT/D, $\mathrm{p}<0.001)$. Entretanto, o desempenho de ambas as 
mãos ao executar a tarefa não foi diferente $(\mathrm{p}>0.05)$ entre si após o pré-teste.

\section{DISCUSSÃO}

O padrão de ganhos iniciais independentes de efetor - observado na melhora de ambas as mãos de PT para RT1 - dando lugar a ganhos dependentes de efetor - observados na melhora do desempenho apenas para a mão não preferida de RT1 para RT2 - é similar aos apontados por outros autores $(19,20,28)$, a despeito da utilização da mão não preferida. Embora nem sempre as assimetrias de preferência se relacionem às assimetrias de desempenho(29), a mão não preferida iniciou a prática com desempenho inferior à mão preferida. Entretanto, houve uma dissipação das diferenças iniciais de desempenho entre as mãos, que pode ser relacionada à evolução para a mão não preferida e à interrupção na melhora do desempenho para a mão preferida, sugerindo que os ganhos após RT2 teriam como característica a dependência efetora. A melhora observada de PT para RT1 na utilização de ambas as mãos também sugere que os ganhos iniciais com a mão não preferida apresentam certo grau de transferência para a mão preferida, ainda que não haja envolvimento dela na prática - tal qual observado no estudo de Korman et al.(13). Assim, é possível argumentar que a sequência de ganhos independentes seguidos de ganhos dependentes de efetor na utilização da mão não preferida é similar, inclusive quanto à escala de tempo do seu aparecimento, à observada na utilização da mão preferida em indivíduos manualmente destros.

Em conjunto, os resultados dos diversos estudos até agora realizados não parecem contradizer a ocorrência de transferência bilateral, ou mesmo a transferência entre diversos grupos musculares efetores reportada anteriormente ${ }^{(12,32)}$. Há, todavia, claras dúvidas quanto aos limites da transferência, particularmente após a progressão da prática, já manifestadas por Jordan ${ }^{(8)}$. Nesse sentido, a prática com a mão não preferida parece apresentar características similares à prática com a mão preferida, tanto no presente estudo como nos realizados por Karni et al. $(9,10)$ e Korman et al. ${ }^{(13)}$.

Diversos modelos têm sido propostos com o objectivo de explicar a progressão de ganhos não específi- cos para ganhos específicos. A partir da associação entre dados comportamentais e de ressonância funcional magnética, Karni et al.(10) sugerem uma progressão de fases distintas de aprendizagem. A primeira fase se constitui de um aprendizado "rápido" inicial, que desencadeia processos neurais que continuam a evoluir por muitas horas após a exposição inicial e formam a base para a consolidação das experiências. A esse período se seguiria uma fase "lenta", de ganhos específicos para o efetor utilizado, na qual há modificações na representação da sequência em substratos neurais críticos para a proficiência motora (como o córtex motor primário). Hirosaka et al.(6) propõem um modelo de redes neurais paralelas para explicar algumas características da aprendizagem de sequências, entre elas a progressão de independência/dependência efetora. Os autores sugerem a existência de dois mecanismos operando em paralelo: um de sequência espacial e outro de sequência motora. Inicialmente, o processo de sequência espacial é responsável pelas conexões entre os mecanismos de cada acção individual, adquirindo a sequência de maneira rápida e flexível com base na atenção e memória de trabalho. O processo é denominado espacial por codificar a sequência em coordenadas visuais, transferíveis para diferentes efetores. Contudo, a informação adquirida no processo é normalmente temporária. O processo de sequência motora, por sua vez, adquire a sequência de forma mais lenta e contínua com a prática de longa duração. A informação adquirida é praticamente permanente, porém específica ao efetor utilizado na prática por ser codificada em coordenadas motoras. Os autores sugerem ainda as áreas neurais responsáveis por cada um dos processos, porém a descrição dos mesmos foge ao escopo deste estudo. Outros modelos foram também propostos para tentar explicar as características da transferência bilateral em tarefas com diferentes níveis de complexidade(27) e a seqüência de independência seguida de dependência efetora ${ }^{(28)}$. Em comum com os modelos descritos acima, os autores destes modelos também sugerem a existência de um nível de codificação independente de efetor e outro dependente de efetor na aprendizagem de habilidades motoras. A proposição de apenas dois mecanismos é possivelmente uma simplificação, como sugerem 
Karni et al.(10), para fornecer uma estrutura conceitual para a descrição e a compreensão dos dados da aprendizagem de habilidades motoras. Diversos processos podem estar simultaneamente activos, mesmo na aprendizagem de relativo curto prazo(26), e tais processos podem ocorrer em uma escala de tempo compatível com a abrangida neste estudo. Não obstante, a convergência dos diversos modelos incita a uma reconsideração de teorias correntes de aprendizagem motora, em que um único nível de representação, independente de efetor, é o único responsável por todos os aspectos da execução do movimento(28).

Em síntese, estudos com diferentes tarefas e metodologias distinguem ganhos iniciais (independentes de efetor) de ganhos posteriores (dependentes de efetor) na aprendizagem de habilidades motoras. $\mathrm{Na}$ transferência bilateral de aprendizagem, observa-se um padrão similar na relação entre o desempenho do membro utilizado na prática e o do membro contralateral. Os resultados do presente estudo mostram que o padrão é semelhante quando a prática é realizada com a mão não preferida (esquerda) de indivíduos destros. Modelos com mais de um nível de representação parecem ser necessários para explicar a progressão da transferência entre diferentes efetores, o que sugere a necessidade da reconsideração de modelos conceituais com um único nível de codificação, de representação unicamente abstracta e independente de efetor.

\section{AGRADECIMENTOS}

Uma versão preliminar deste trabalho foi apresentada no congresso denominado "Progress in Motor Control VI", organizado pela ISMC (International Society for Motor Control). Ulysses Okada de Araujo agradece o apoio financeiro oferecido pelo Conselho Nacional de Desenvolvimento Científico e Tecnológico (CNPq) para o desenvolvimento das actividades de pós-graduação. Os autores agradecem as observações do(s) revisor(es) anônimo(s) referentes à versão anterior deste manuscrito.

\section{CORRESPONDÊNCIA}

Ulysses Okada de Araujo

Laboratório de Comportamento Motor

Escola de Educação Física e Esporte - USP

Av. Prof. Mello Moraes, 65, Butantã, São Paulo

SP, CEP 05508-900, Brasil

E-mail: ulokada@usp.br 


\section{REFERÊNCIAS}

1. Ammons RB, Ammons CH (1951). Bilateral transfer of rotary pursuit skill. Am Psychol 6(7): 298.

2. Bapi RS, Doya K, Harner AM (2000). Evidence for effector independent and dependent representations and their differential time course of acquisition during motor sequence learning. Exp Brain Res 132(2): 149-162.

3. Buchanan JJ (2004). Learning a single limb multi-joint coordination pattern: The impact of a mechanical constraint on the coordination dynamics of learning and transfer. Exp Brain Res 156(1): 39-54.

4. Cook TW (1933). Studies in cross education II. Further experiments in mirror tracing the starshaped maze. J Exp Psychol Gen 16(5): 679-700.

5. Grafton ST, Hazeltine E, Ivry RB (2002). Motor sequence learning with the nondominant left hand: A PET functional image study. Exp Brain Res 146(3): 369-378.

6. Hirosaka O, Nakahara H, Rand MK, Sakai K, Lu XF, Nakamura K, Myiachi S, Doya K (1999) Parallel neural networks for learning sequential procedures. Trends Neurosci 22(10): 464-471.

7. Japkise KC, Negash S, Howard JH, Howard DV (2003). Intermanual transfer of procedural learning after extended practice of probabilistic sequences. Exp Brain Res 148(1): 38-49.

8. Jordan MI (1995). The organization of action sequences: Evidence from a relearning task. J Mot Behav 27(2): 179192.

9. Karni, A, Meyer G, Jezzard P, Adams MM, Turner R, Ungerleider LG (1995). Functional MRI evidence for adult motor cortex plasticity during motor skill learning. Nature 377(6545): 155-158.

10. Karni A, Meyer G, Rey-Hipolito C, Jezzard P, Adams MM, Turner R, Ungerleider LG (1998). The acquisition of skilled motor performance: Fast and slow experience-driven changes in primary motor cortex. Proc Natl Acad Sci U S A 95(3): 861-868.

11. Kelso JAS, Zanone P-G (2002). Coordination dynamics of learning and transfer across different effector systems. $J$ Exp Psychol Hum Percept Perform 28(4): 776-797.

12. Keele SW, Jennings P, Jones S, Caulton D, Cohen A (1995). On the modularity of sequence representation. $J$ Mot Behav 24(1): 17-30.

13. Korman N, Raz N, Flash T Karni A (2003). Multiple shifts in the representation of a motor sequence during the acquisition of skilled performance. Proc Natl Acad Sci 100(21): 12492-12497.

14. Kumar S, Mandal, MK (2005). Bilateral transfer of skill in left- and right-handers. Laterality 10(4): 377-344.

15. Mackay DG (1982). The problems of flexibility, fluency and speed-accuracy trade-off in skilled behavior. Psychol Rev 89(5): 483-506.
16. Magill, RA (2001). Motor learning: concepts and applications (6th ed.). Boston: McGraw-Hill.

17. Oldfield RC (1933) The assessment and analysis of handedness: The Edinburgh inventory. Neuropsychologia 9(1): 97-113.

18. Osman M, Bird G, Heyes C (2005). Action observation supports effector-dependent learning of finger movement sequences. Exp Brain Res 165(1): 19-27.

19. Park J-H, Shea CH (2003). Effect of practice on effector independence. J Mot Behav 35(1): 33-40.

20. Park J-H, Shea CH (2005). Sequence learning: Response structure and effector transfer. Q J Exp Psychol A 58(3): 387-419.

21. Rose DJ, Christina RW (2006) A multilevel approach to the study of motor control and learning (2nd ed.). San Francisco: Pearson/Benjamin Cummings.

22. Rosenbaum DA, Kenny SB, Deer MA (1983). Hierarchical control of rapid movement sequences. J Exp Psychol Hum Percept Perform 9(1): 86-102.

23. Schmidt RA, Lee TD (1999). Motor control and learning: $a$ behavioral emphasis (3rd ed.). Champaign, IL: Human Kinetics.

24. Schmidt RA (1975). A schema theory of discrete motor skill learning. Psychol Rev 82(4): 225-260.

25. Schöner G, Zanone P-G, Kelso JAS (1992). Learning as change in coordination dynamics: Theory and experiment. J Mot Behav 24(1): 29-48.

26. Smith MA, Ghazizadeh A, Shadmehr R (2006). Interacting adaptative processes with different timescales underlie short-term motor learning. PLoS Biol 4(6): 1035-1043.

27. Teixeira LA (2006). Intermanual transfer of timing control between tasks holding different levels of motor complexity. Laterality 11(1): 43-56.

28. Vangheluwe S, Suy E, Wenderoth N, Swinnen SP (2006). Learning and transfer of bimanual multifrequency patterns: Effector-independent and effector specific levels of movement representation. Exp Brain Res 170(4): 543-554.

29. Vasconcelos O (2004). Preferência lateral e assimetria motora funcional: uma perspectiva de desenvolvimento. In: Barreiros J, Godinho M, Melo F, Neto C (eds.). Desenvolvimento e aprendizagem: perspectivas cruzadas. Lisboa: Edições FMH, 67-93.

30. Verwey WB, Clegg BA (2005). Effector-dependent sequence learning in the serial RT task. Psychol Res 69(4): 242251.

31. Verwey WB, Wright DL (2004). Effector-independent and effector-dependent learning in the discrete sequence production task. Psychol Res 68(1): 64-70.

32. Wright CE (1990). Generalized motor programs: Reexamining claims of effector independence in writing. In: Jeannerod M (ed.) Attention and performance XIII. Hillsdale, NJ: Erlbaum, 294-320. 